\title{
LA CIRCOLAZIONE DEL MARMO NELL'ADRIATICO DURANTE LA TARDA ANTICHITÀ
}

\author{
YURI A. MARANO
}

UDC: 903.25:552.46(262.3)"04/05"

Review

Manuscript received: 05. 11. 2015.

Revised manuscript accepted: 18. 03. 2016.

DOI: 10.1484/J.HAM.5111340

Y. A. Marano
Via Jesi 267
60027 Osimo (Ancona)
Italia

The circulation of marble artefacts (primarily architectural elements and liturgical furnishings), produced in the quarries and workshops in the island of Prokonnesos, is one of the most distinctive phenomena in the 5th and 6th centuries. Apart from Constantinople, some of the richest collections of such materials have been found along the shores of the Adriatic Sea. So far, modern scholars believe that Ravenna acted as the redistribution centre of these materials. However, from the analysis of architectural and historical contexts of the period, it is apparent that the presence of prokonnesian marble at sites on the Adriatic coast marks the intervention of the imperial and Church authorities in sites of political, strategic and religious relevance. Seemingly marble was directly imported from Constantinople rather than passing through Ravenna, and the lay and ecclesiastic patrons were first engaged in its circulation.

Key-words: Adriatic Sea, marble artefacts, architectural elements, liturgical furnishings, lay and ecclesiastic patronage.

All'incirca trent'anni fa, John Clayton Fant ha definito il commercio romano del marmo "an improbabile phenomenon". È noto, infatti, che nell'Antichità il trasporto su lunghe distanze della pietra richiedesse considerevoli investimenti economici e logistici, risultando irrazionale in una regione come il Mediterraneo, dove materiali lapidei di buona qualità sono disponibili pressoché ovunque. Questa “irrazionalità" alimentava però il prestigio del marmo e delle pietre colorate, rendendole simboli di potere e ricchezza direttamente associati alla sfera imperiale. Sebbene gli imperatori romani non abbiano mai esercitato un monopolio assoluto sul marmo, è indubbio che le esigenze della committenza ufficiale abbiano impresso uno stimolo decisivo alla sua produzione e consumo ${ }^{2}$. Tale legame tra potere imperiale e utilizzo del marmo si rafforzò ulteriormente nel corso della tarda Antichità, in concomitanza con il declino dell'evergetismo privato. Tra il IV e il VI secolo, l'impiego di grandi quantità di marmo caratterizzò quasi esclusivamente i progetti edilizi promossi dagli imperatori o dai rappresentanti delle più alte gerarchie laiche ed ecclesiastiche. Non a caso, la scultura costantinopolitana, diffusa in località di particolare rilievo politico, strategico e religioso ${ }^{3}$, può essere utilizzata per ricostruire le relazioni tra la capitale e le province dell'impero ${ }^{4}$. È da questa prospettiva che il presente contributo intende analizzare la diffusione di questi materiali nell'Adriatico (fig. 1), situandola nel contesto storico del V e VI secolo e riconsiderando il ruolo di Ravenna quale presunto centro di diffusione dei prodotti e dei modelli artistici elaborati a Costantinopoli.

\section{VENETIA}

Se si escludono le spoglie della Quarta Crociata giunte nei territori della Serenissima a partire dal 1204, la Venetia ha restituito un numero limitato di testimonianze relative alla circolazione di marmi di origine costantinopolitana. Una parziale eccezione è rappresentata da Aquileia, città che pare avere mantenuto il proprio ruolo di caput provinciae oltre il sacco attilano, attirando l'attenzione delle autorità bizantine ancora all'indomani della guerra gotica5. Qui, tra i materiali di importazione più antichi si segnala un erratico capitello ionico in marmo di Thasos della fine del IV - inizi del V secolo, appartenente a una tipologia non altrimenti attestata nell'Adriatico, ma la cui esportazione

${ }^{1}$ J.C. FANT, The Roman Emperors in the Marble Business: Capitalists, Middlemen, and Philanthropists, in Classical Marble: Geochemistry, Technology, Trade, a cura di N. Herz e M. Waelkens, Dordrecht-Boston-London, 1988, p. 147-158, in part. p. 147.

${ }^{2}$ Sulla produzione e il consumo del marmo e delle pietre colorate nel mondo romano, cfr. P. PENSABENE, I marmi nella Roma antica, Roma, 2014, e B. RUSSELL, The Economics of Roman Stone Trade, Oxford, 2013.

${ }^{3}$ Sulla diffusione della scultura costantinopolitana nel Mediterraneo tardoantico, J.-P. SODINI, C. BARSANTI, A. GUIGLIA GUIDOBALDI, La sculpture architecturale en marbre au Vie siècle à Constantinople et dans les régions sous influence constantinopolitaine, in Acta XIII Congressus Internationalis Archaeologiae Christianae (Split-Poreč, 25.9 - 1.10) (Studi di Antichità Cristiana 54), Città del Vaticano, 1998, p. 301-376; sulla circolazione di questi materiali nell'Adriatico, cfr. P. PENSABENE, C. BARSANTI, Reimpiego e importazione di marmi nell'Adriatico adriatico e bizantino, in La cristianizzazione dell'Adriatico, a cura di G. Cuscito (Antichità Altoadriatiche 66), Trieste, 2008, p. 455-490.

${ }_{4}^{4}$ Sull'utilizzo della cultura materiale e delle produzioni artistiche per lo studio delle relazioni tra Costantinopoli e le province dell'impero bizantino, si veda M. MUNDELL MAGNO, The Centre In and Beyond the Periphery: Material Culture in the Early Byzantine Empire, in Byzantina-Metabyzantina. La périphérie dans les temps et dans l'espace. Actes de la $6^{e}$ Séance plénière du XXe Congrès international des Études byzantines (Collège de France - Sorbonne, Paris 19-25 Août 2002), a cura di P. Odorico, Paris 2003, p. 119-140.

${ }_{5}^{5}$ Y.A. MARANO, Urbanesimo e storia ad Aquileia tra Ve VI secolo d.C., in L'architettura privata ad Aquileia in età romana, a cura di J. Bonetto e M. Salvadori (Atti del convegno di studio, Padova, 21-22 febbraio 2011), Padova, 2012, p. 571-590, in part. p. 579-590 (con bibliografia precedente). 


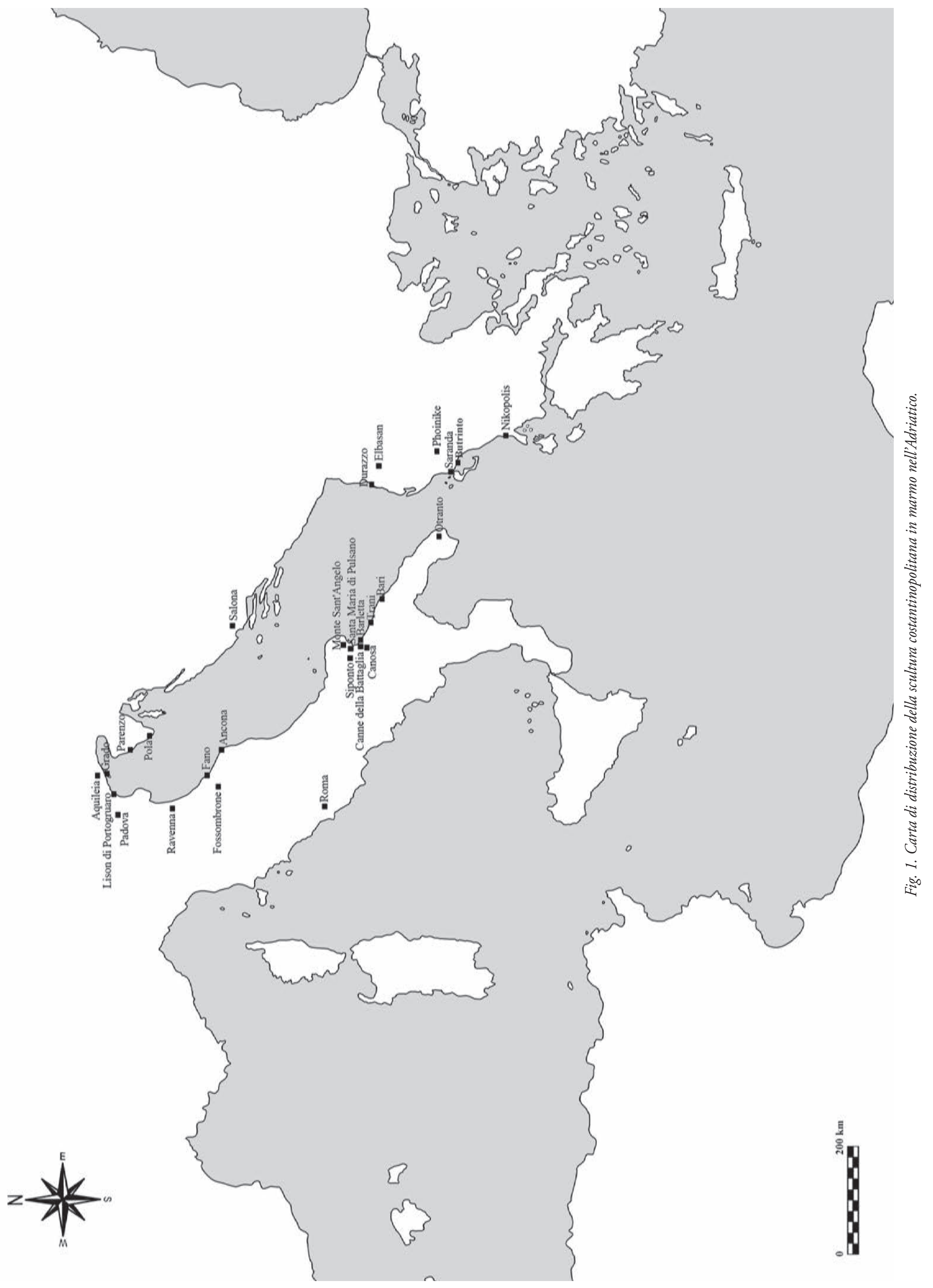

Y. A. Marano: La circolazione del marmo... 167 


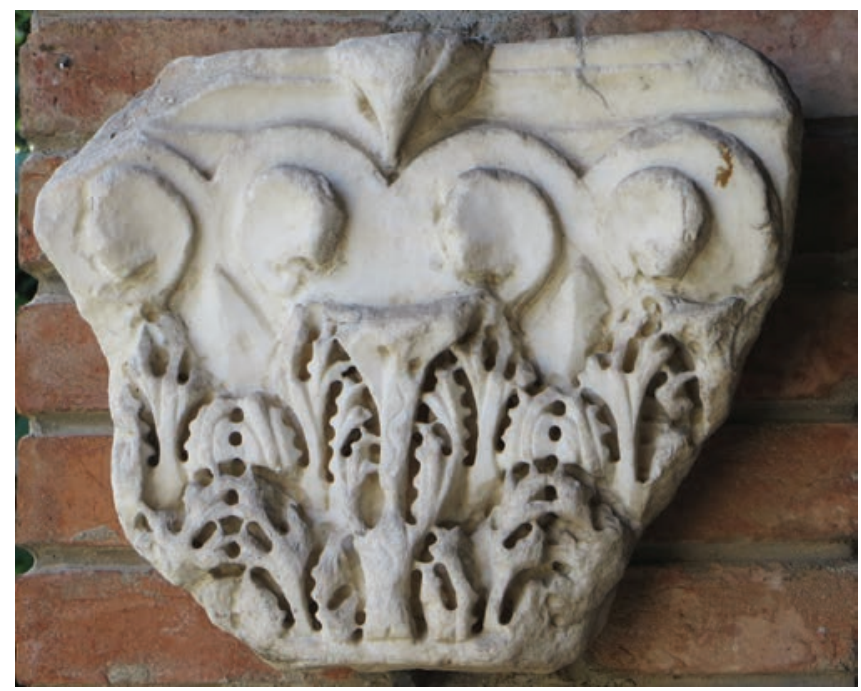

Fig. 2. Aquileia, Museo Archeologico Nazionale: capitello di lesena corinzio ad acanto finemente dentellato (foto autore).

nel Mediterraneo occidentale trova riscontro a Roma e a Ostia $^{6}$. Di fattura microasiatica è invece un capitello di lesena corinzio ad acanto finemente dentellato del V/VI secolo (fig. 2), anch'esso fuori contesto, ma confrontabile con esemplari di reimpiego nella basilica romana di Santa Maria Antiqua ${ }^{7}$. Allo stesso periodo si datano anche le sculture di arredo architettonico e liturgico della cattedrale e della chiesa di Monastero, il cui rinnovamento implicò l'utilizzo di materiali di origine o di imitazione costantinopolitana ${ }^{8}$. Per quel che concerne le produzioni locali, un elemento interessante è rappresentato da un capitello-imposta ionico in calcare d'Aurisina (fig. 3), verosimilmente proveniente da un edificio di culto, come suggerisce la croce latina a estremità patenti che ne decora entrambe le facce principali甲. Il capitello può essere avvicinato a una nutrita serie di elementi analoghi presenti lungo le coste della Dalmazia, delle Marche, della Puglia e dell'Epiro, i cui prototipi vanno individuati nell'Egeo. Del resto, la ricezione di modelli orientali da parte delle officine aquileiesi della tarda Antichità è testimoniata da alcuni capitelli corinzi e basi d'acanto in calcare, che si caratterizzano per la me-

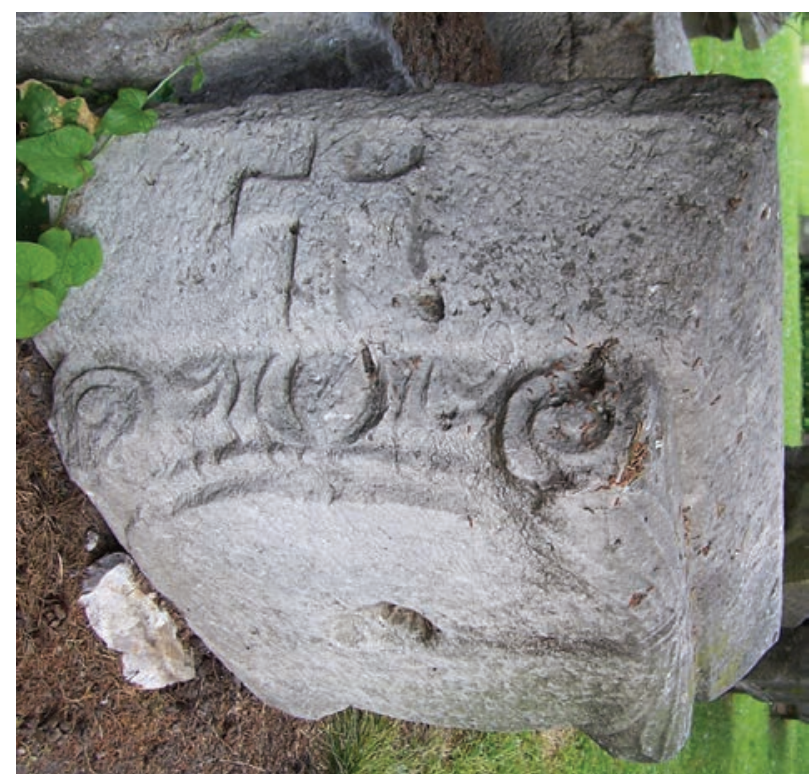

Fig. 3. Aquileia, Museo Archeologico Nazionale: capitello-imposta ionico (foto autore).

scolanza di modelli costantinopolitani e di modelli locali di tradizione tardorepubblicana e augustea ${ }^{10}$.

Un quadro molto simile caratterizza anche Grado, dove le basiliche di S. Eufemia e di S. Maria delle Grazie custodiscono capitelli in marmo di tipologia e cronologia differenti, la cui attribuzione alle fasi più antiche di questi edifici è però tutt'altro che sicura ${ }^{11}$. Lo studio degli arredi liturgici permette, invece, di ricostruire l'attività di botteghe locali, i cui prodotti si caratterizzano per il ricorso a motivi decorativi di chiara ascendenza costantinopolitana, recepiti e reinterpretati tramite la mediazione di Ravenna ${ }^{12}$.

Sulla terraferma, la presenza di sculture d'importazione appare sporadica. Non vi sono elementi a sostegno dell'appartenenza all'arredo liturgico della basilica paleocristiana di Concordia Sagittaria delle tre arcate di ciborio conservate presso la chiesa parrocchiale della Natività di Maria a Lison di Portogruaro (Venezia) ${ }^{13}$. Non solo lo scarto qualitativo di questi elementi rispetto ai plutei di manifattura locale rinvenuti all'interno dell'edificio di culto è netto, ma l'iscri-

\footnotetext{
${ }^{6}$ Sulla produzione di questi elementi, cfr. J.J. HERRMANN, J.-P. SODINI, Exportations de marbre thasien è l'époque paléochrétienne : le cas des chapiteaux ioniques, in Bulletin de Correspondance Hellénique 101, p. 475-511.

7 P. PENSABENE, Il reimpiego ad Aquileia: problematiche aperte, in Riuso di monumenti e reimpiego di materiali antichi in età postclassica: il caso della Venetia, a cura di G. Cuscito (Antichità Altoadriatiche 74), Trieste, 2012, p. 85-102, in part. p. 92. Sul tipo, cfr. J. KRAMER, Korinthische Pilasterkapitelle in Kleinasien und Konstantinopel. Antike und spätantike Werkstattgruppen (Istanbuler Mitteilungen 39), Tübingen, 1994.

${ }^{8}$ M. BUORA, Testimonianze di scultura di età gota e bizantina nella basilica di Aquileia e nella chiesa di Monastero. Nuove considerazioni, in Arheološki vestnik 66, 2015, p. 205-234, in part. p. 209-222.

${ }_{9}^{9}$ Già segnalato presso il battistero, l'elemento è oggi conservato nei giardini del Museo Archeologico Nazionale (S. TAVANO, Sculture aquileiesi e gradesi inedite, in Memorie Storiche Forogiuliesi 51, p. 95-117, in part. p. 99-101). Di forma tronconica, il capitello mostra su ambo le facce una croce latina a estremità patenti e da un kyma sul quale è un ovulo inquadrato da palmette tra volute spiraliformi; sul balaustro sono, invece, tre foglie lanceolate trattenute centralmente da una fascia, mentre l'astragalo è decorato da perline e fusarole. Queste caratteristiche permettono di assimilare l'esemplare aquileiese al tipo II, 2 della classificazione di V. VEMI, Les chapiteaux ionique à imposte de Grèce à l'époque paléochrétienne (Bulletin de Correspondance Hellenique - Supplément 17), Athènes-Paris 1989, p. 19-21.

${ }^{10}$ P. PENSABENE, Il reimpiego... op. cit., p. 92-93; M. PILUTTI, I capitelli di navata della cattedrale di Santa Eufemia e della chiesa di Santa Maria delle Grazie a Grado, in Annali della Scuola Normale Superiore di Pisa, s. IV, vol. 9/2, 2004, 269-304, in part. p. 298.

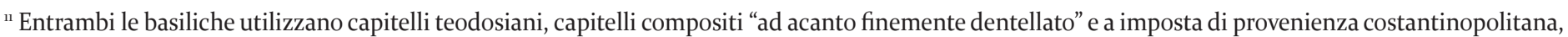
oltre a fusti di colonna in marmi bianchi e colorati (cipollino, portasanta, granito, proconnesio, bianco e nero d'Aquitania): PILUTTI, I capitelli... op. cit. p. 269-304; A. TAGLIAFERRI, Le diocesi di Aquileia e Grado (Corpus della Scultura Altomedievale 10), Spoleto 1981, p. 339-425.

${ }^{12}$ A. TERRY, The Early Christian Sculpture at Grado: A Reconsideration, in Gesta 26, 1987, p. 93-112.

${ }^{13}$ M. BONFIOLI, Tre arcate protobizantine a Lison di Portogruaro (Ricuperi bizantini in Italia 1), Roma, 1979. L'ipotesi dell'appartenenza delle arcate all'arredo liturgico della basilica di Concordia Sagittaria è stata avanzata da P. CROCE DA VILLA, Concordia dal IV al IX secolo d.C., Fiume Veneto, 1989, p. 231-232, in part. 237-238, e ripresa da G. CANTINO WATAGHIN, Monasterium... in locum qui vocatur Sexto. L'archeologia per la storia dell'abbazia di Santa Maria di Sesto, in L'abbazia di Santa Maria di Sesto fra archeologia e storia, a cura di G.C. Menis e A. Tilatti, Fiume Veneto, 1999, p. 3-51, in part. p. 34.
} 


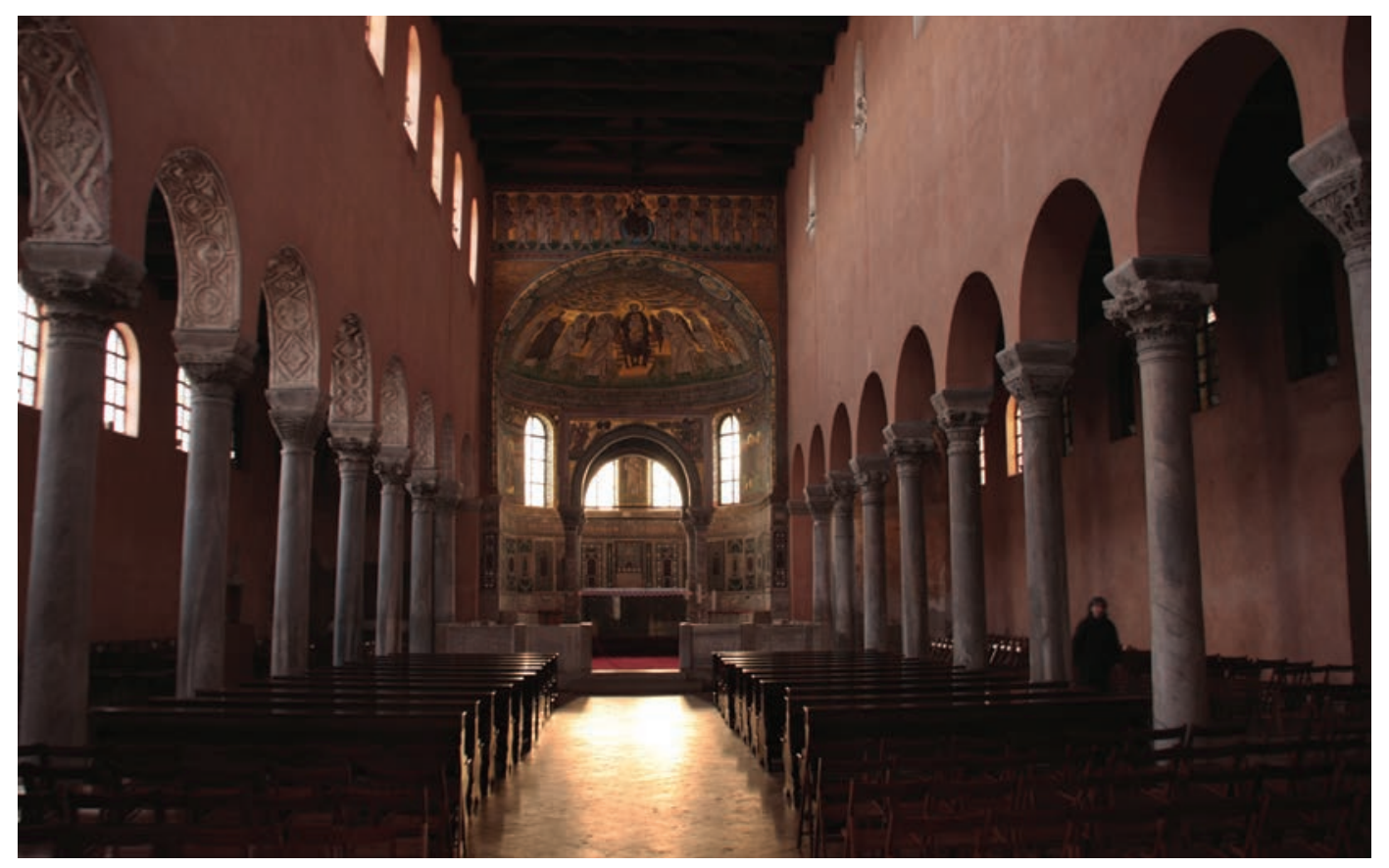

Fig. 4. Poreć, basilica eufrasiana (foto M. Jurković).

zione greca che ne ricorda la dedicazione da parte del sinator Stefano, un membro delle scholae palatinae (la guardia personale dell'imperatore d'Oriente), mal si accorda con un loro arrivo in antico nei territori della Venetia ${ }^{14}$. È dunque probabile che le arcate siano giunte a Lison attraverso il mercato antiquario veneziano di età moderna ${ }^{15}$.

Un insieme di notevole interesse è rappresentato dalla pergula in marmo del sacello di S. Prosdocimo a Padova, eretta entro il primo venticinquennio del VI secolo dal vir clarissimus et inlustris praefecto praetorio adque patricius Opilione $^{16}$. Assai più modesto un frammento di balaustra in marmo lunense dalla cattedrale di Vicenza che, da riferirsi quasi sicuramente a un ambone a doppia rampa scalare, dimostra l'adozione nell'entroterra veneto di un tipo di arredo proprio di Costantinopoli, diffuso, oltre che nelle province balcaniche dell'impero, anche a Ravenna e nell'area adriatica $^{17}$.

\section{ISTRIA E DALMAZIA}

Rispetto alla Venetia, sulle coste nordorientali dell'Adriatico il quadro delle importazioni di scultura costantinopolitana è assai più ricco e articolato.

In Istria, la cattedrale di Poreč, costruita dal vescovo Eufrasio attorno alla metà del VI secolo, conserva uno straordinario corpus di materiali ${ }^{18}$, tra cui sono quaranta capitelli di diversa tipologia (compositi ${ }^{19}$, bizonali ${ }^{20} \mathrm{e}$ "a cesto" ${ }^{21}$ ), tutti in marmo proconnesio e con precisi confronti a Costantinopoli, a Ravenna, a Cartagine e in altri centri del Mediterraneo bizantino (fig. 4). A questi si aggiungono diciotto fusti di colonna e basi di tipo attico ${ }^{22}$, quattro portali in marmo (di cui uno destinato all'episcopio), quattro pietre d'imposta e i pilastrini di una recinzione presbiteriale, la cui provenienza dalle cave del Proconneso è certificata da masons' marks in lettere greche ${ }^{23}$. I plutei ${ }^{24}$ e l'ambone ${ }^{25}$ della cattedrale

\footnotetext{
${ }^{14}$ A questo proposito, non può essere accolta nemmeno la proposta di G. Ravegnani, Soldati di Bisanzio nelle epigrafi del VI secolo, in Studi in ricordo di Fulviomaria Broilo, a cura di G. Cresci Marrone e A. Pistellato (Atti del Convegno, Venezia, 14-15 ottobre 2005), Padova, 2007, p. 532-530, in part. 526-527, che ritiene Stefano giunto a Concordia assieme ai contingenti imperiali distaccati in Italia da Tiberio II (578-582). Un'ipotesi smentita dalla datazione delle arcate ai primi decenni del VI secolo.

${ }^{15}$ M. BONFIOLI, Tre arcate... op. cit., p. 32-34 e 89-95.

${ }^{16}$ Tale datazione si basa sul fatto che l'iscrizione di dedica non faccia alcuna menzione del consolato, carica che Opilione rivestì dopo il 524 d.C. (M. SANNAZARO, Padova, in Il Veneto nel medioevo. Dalla "Venetia" alla marca veronese, a cura di A. Castagnetti e G.M. Varanini, Verona, 1989, p. 220-244, in part. p. 240-242.

${ }^{17}$ E. NAPIONE, La diocesi di Vicenza (Corpus della Scultura Altomedievale 14), Spoleto, 2001, p. 246-247.

${ }^{18}$ E. RUSSO, Sculture del complesso eufrasiano di Parenzo, Napoli, 1992; A. TERRY, The Sculpture at the Cathedral of Eufrasius in Poreč, in Dumbarton Oaks Papers, 42, 1988, p. 13-64.

${ }_{19}$ RUSSO , Sculture... op. cit., p. 38-39, 41, 46-47, 52-55, 6o-61; sui capitelli, cfr. anche TERRY, The Sculpture ... op. cit., p. 18-25.

${ }^{20}$ RUSSO , Sculture... op. cit., p. 39-45, 53-54.

${ }^{21}$ RUSSO , Sculture... op. cit., p. 42-45, 47-50, 144-147.

${ }^{22}$ L'importazione di diciotto fusti di colonna dalle cave del Proconneso evidenzia l'impegno e le risorse profusi da Eufrasio della costruzione e nella decorazione della sua basilica: il numero è infatti di poco inferiore a quello delle ventiquattro colonne in marmo proconnesio delle basiliche di Sant'Apollinare Nuovo a Ravenna (E. PENNI IACCO, La basilica di Sant'Apollinare Nuovo a Ravenna attraverso i secoli, Bologna, 2004, p. 33-34) e di Sant'Apollinare in Classe (F.W. DEICHMANN, Ravenna. Hauptstadt des spätantiken Abendlandes. Kommentar, 2. Teil, Wiesbaden, 1974, p. 241).

${ }^{23}$ RUSSO, Sculture... op. cit., p. 161-168; TERRY, The Sculpture... op. cit., p. 39-43

${ }^{24}$ Sostenuta da TERRY, The Sculpture... op. cit., p. 35-39, l'attribuzione dei plutei a maestranze locali è contestata da RUSSO, Sculture... op. cit. p. 130, che li ritiene opera di maestranze greco-costantinopolitane di livello non eccelso.

${ }_{25}$ Contraddistinto da una doppia rampa scalare e da una piattaforma ottagonale sorretta da colonnine, l'ambone combina le caratteristiche degli amboni costantinopolitani con quelle di alcuni arredi rinvenuti in Grecia. Un particolare che trova confronto tanto a Grado quanto in Dalmazia (P. CHEVALIER, L'ambon à Poreč et Istrie, in Hortus Artium Medievalium, 1, 1995, p. 126-133.
} 


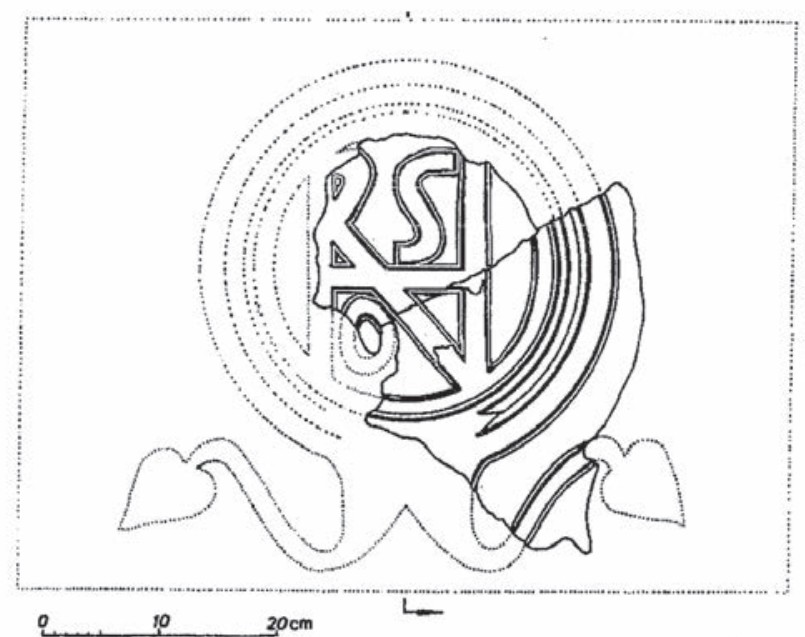

Fig. 5. Salona, basilica cruciforme: pluteo con monogramma del vescovo Onorio II (da Chevalier-Meztger 1994).

sono anch'essi in marmo proconnesio, ma vanno assegnati a maestranze locali.

Tre plutei a serti lemniscati facevano parte dell'arredo liturgico della cattedrale di Pola, al cui battistero va ricondotto un capitello bizonale con colombe angolari e sottostante canestro vitineo, oggi conservato al museo archeologico della città $^{26}$. Due frammenti di plutei provengono anche dalla basilica di S. Maria Formosa ${ }^{27}$, fondata nella sua città natale dall'arcivescovo Massimiano di Ravenna (546-554 d.C) ${ }^{28}$.

A Salona (Dalmazia), il periodo immediatamente successivo alla riconquista giustinianea fu segnato da un'intensa attività edilizia, promossa dai vescovi locali nelle chiese della città e del suburbio, il cui restauro implicò anche il rinnovamento degli arredi liturgici ${ }^{29}$. Nel complesso episcopale, Onorio II (527-547) dotò la basilica cruciforme di una nuova recinzione presbiteriale, facendo realizzare una serie di plutei con il proprio monogramma ${ }^{30}$ (fig. 5). Nella basilica di Manastirine sono, invece, due transenne in marmo proconnesio lavorate a giorno con archetti a tutto sesto ornati da conchiglie e sorretti da colonnine, le cui dimensioni si adattano perfettamente a quelle del presbiterio, disassato rispetto all'edificio di culto. Un particolare, questo, che ne fa supporre la realizzazione in loco da maestranze costantinopolitane, cui vanno attribuite anche le analoghe transenne della basilica urbana ${ }^{31}$.

\section{MARCHE}

Nel territorio delle Marche, il duomo di S. Ciriaco ad Ancona reimpiega un numero cospicuo di capitelli corinizi, capitelli ionici-imposta, fusti di colonna e imposte costantinopolitani ${ }^{32}$, verosimilmente pertinenti alla basilica paleocristiana di S. Lorenzo (V/VI secolo), i cui resti sono stati individuati sotto la fabbrica romanica ${ }^{33}$. Dell'arredo liturgico di S. Lorenzo faceva probabilmente parte anche l'ambone a doppia rampa scalare, il cui parapetto, decorato da un motivo a quadrati concentrici con vertici a foglia di cuore e fiorone quadrigigliato centrale, è esposto al Museo Diocesano34 (fig. 6).

Questi materiali non sono una presenza isolata: le indagini condotte in passato presso la basilica di S. Maria alla Piazza, ricostruita in età giustinianea, hanno permesso il recupero di un capitello corinzio e di due pilastrini decorati sulla fronte da un riquadro modanato, di cui uno con terminazione a bulbo appuntito e uno in origine pertinente

${ }^{26}$ T. ŠEPAROVIĆ, "Capitello", in Bizantini, Croati, Carolingi. Alba e tramonto di regni e imperi, a cura di C. Bertelli et Alii (Catalogo della mostra, Brescia), Milano, 2001, p. 286.

${ }_{27}$ Ž. UJČIĆ, La basilica paleocristiana di Santa Maria Formosa a Pola, Pola, 2005, p. 39.

${ }^{28}$ Agnelli, Liber pontificalis ecclesiae ravennatis 76, ed. d. Mauskopf, Corpus Christianorum Series Latina. Continuatio Medievalis, 199, Turnhout, 2006, p. 243: [Maximianus] Aedificavit que ecclesiam beatae Mariae in Pola quae vocatur Formosa, unde diaconus fuit, mira pulchritudine, et diversis ornavit lapidibus. ${ }^{29}$ J. JELIČIC-RADONIĆ, The Salonitan Cultural Circle of Justinian's Time, in Acta XIII Congressus Internationalis Archaeologiae Christianae (Split-Poreč, 25.9 - 1.10) (Studi di Antichità Cristiana 54), Città del Vaticano, 1998, p. 1023-1036; P. CHEVALIER, J. MARDEŠIĆ, Le group épiscopal de Salone au VieVIIe siècles. Urbanisme et vie quotidienne, in Mélanges de l'École française de Rome - Moyen Âge, 120, 2008, p. 227-238.

${ }^{30}$ Sulla risistemazione del settore presbiteriale, CHEVALIER, MARDEŠIĆ, Le groupe épiscopal... op. cit. p. 238-239; sui plutei, P. CHEVALIER, C. METZGER, Plaques de chancel en marbre, in Salona I. Sculpture architecturale. Recherches archéologiques franco-croates, a cura di N. Duval, E. Marin, C. Metzger (Collection de l'École française de Rome 194), Rome 1994, p. 238-247 ; sul monogramma di Onorio II, N. DUVAL, J.-P. CAILLET, Monogramme de l'évêque Honorius, sur un plaque de marbre (verso 500, o entre 527 et 547), in Salona IV. Inscriptions de Salone chrétienne, a cura di E. Marin (Collection de l'École française de Rome 194/4, Rome-Split 2010), p. 219-224. L'uso di apporre monogrammi su plutei è attestato da Paolo Silenziario, che menziona gli esemplari della Santa Sofia siglati con il nome di Giustiniano e Teodora. A livello archeologico, il confronto più diretto per i plutei di Salona è offerto dai plutei della basilica di S. Clemente a Roma, su cui compare il monogramma di papa Giovanni II (533-535 d.C.). A questi si possono affiancare una lastra proveniente dalle Terme Occidentali di Kos, un pluteo con monogramma a legatura quadrata entro un clipeo modanato dalla basilica di S. Demetrio a Salonicco, un frammento con monogramma a legatura cruciforme già nella S. Sofia di Nicea/Iznik (F. GUIDOBALDI, C. BARSANTI, A. GUIGLIA GUIDOBALDI, San Clemente. La scultura del VI secolo (San Clemente Miscellany IV/2), Roma 1992, p. 54 e 154-55) e i plutei della Katapoliani di Paros (A.

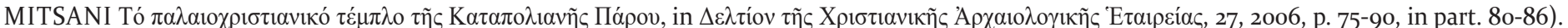
Dall'area del complesso episcopale di Salona provengono anche quattro capitelli bizonali di elevata qualità (I. NIKOLAJEVIĆ, Salona cristiana au VIe et VIIe s., in Disputationes Salonitanae 1970, Split, 1975, p. 91-95; SODINI, BARSANTI, GUIGLIA GUIDOBALDI, La sculpture architecturale... op. cit., p. 325). ${ }^{31}$ C. METZGER, Chancels à colonnettes, in Salona I. Sculpture architecturale. Recherches archéologiques franco-croates, a cura di N. Duval, E. Marin, C. Metzger (Collection de l'École française de Rome 194), Rome 1994, p. 236-238.

${ }^{32}$ C. BARSANTI, Testimonianze bizantine in Ancona. Le spoglie paleocristiane del San Ciriaco, in Atti del V Congresso Nazionale di Archeologia Cristiana (Pesaro - Ancona, 19-23 settembre 1983), Firenze, 1983, p. 387-404.

${ }_{33}$ La basilica di S. Lorenzo è stata datata al passaggio tra il V e il VI secolo da L. PANI ERMINI, La chiesa di San Lorenzo, in San Ciriaco. La cattedrale di Ancona. Genesi e sviluppo, a cura di M.L. Polichetti, Milano, 2003, p. 95-115, in part. 104-108, 111-112, mentre M.C. PROFUMO, Aspetti e problemi della cristianizzazione dell'area picena, in La cristianizzazione dell'Adriatico, a cura di G. Cuscito (Antichità Altoadriatiche 66), Trieste, 2008, p. 151-192, in part. p. 158-162, ne abbassa la cronologia di qualche decennio, collocandola nel pieno VI secolo.

${ }^{34}$ C. BARSANTI, Mosaici pavimentali paleocristiani in Ancona: alcune riflessioni, in Atti del II Colloquio dell'Associazione Italiana per lo Studio e la conservazione del mosaico (Roma, 5-7 dicembre 1994), a cura di F. Guidobaldi e I. Bragantini, Bordighera, 1995, p. 181-200, in part. 186. 


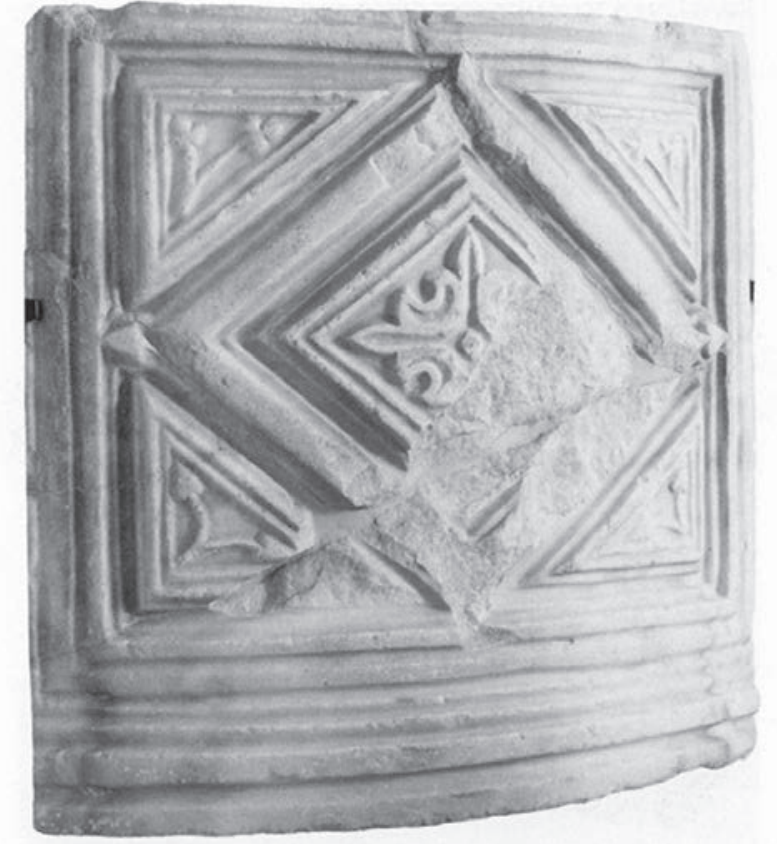

Fig. 6. Ancona, Museo Diocesano: parapetto d'ambone (da Polverari 1992).

a una colonnetta ${ }^{35}$. Altri materiali costantinopolitani erano conservati nella chiesa di S. Pellegrino, dove un pluteo con croce a braccia patenti sigillava il sarcofago altomedievale dei santi Pellegrino e Flaviano ${ }^{36}$; nello stesso edificio, si trovava anche l'urna di san Dasio, prodotto di officine asiane del II/ III secolo e sottoposto a rilavorazione nel VI secolo, quando sul coperchio furono scolpite tre croci latine a braccia patenti, tra cui si dispone l'iscrizione greca che ricorda la traslazione delle reliquie del santo da Durostorum (l'attuale Silistra, in Bulgaria) ${ }^{37}$.

Nell'entroterra, le collezioni del Museo Civico A. Vernarecci di Fossombrone (Pesaro-Urbino) comprendono un interessante pilastrino di recinzione presbiteriale in pietra locale, fedele replica dei modelli costantinopolitani del periodo giustinianeo ${ }^{38}$ (fig. 7). Decorato sulla faccia anteriore da un motivo a tralcio vitineo, esso richiama i pilastrini in marmo proconnesio della recinzione presbiteriale della basilica di San Clemente a Roma, che servirono da modello anche per gli esemplari del complesso di San Calogero ad Albenga (Savona) 39 . Molto probabilmente, questi elementi furono realizzati, in marmo lunense e "in tono minore", da uno o più scultori che, già attivi sul cantiere di S. Clemente, potrebbero avere raggiunto la Liguria risalendo la costa

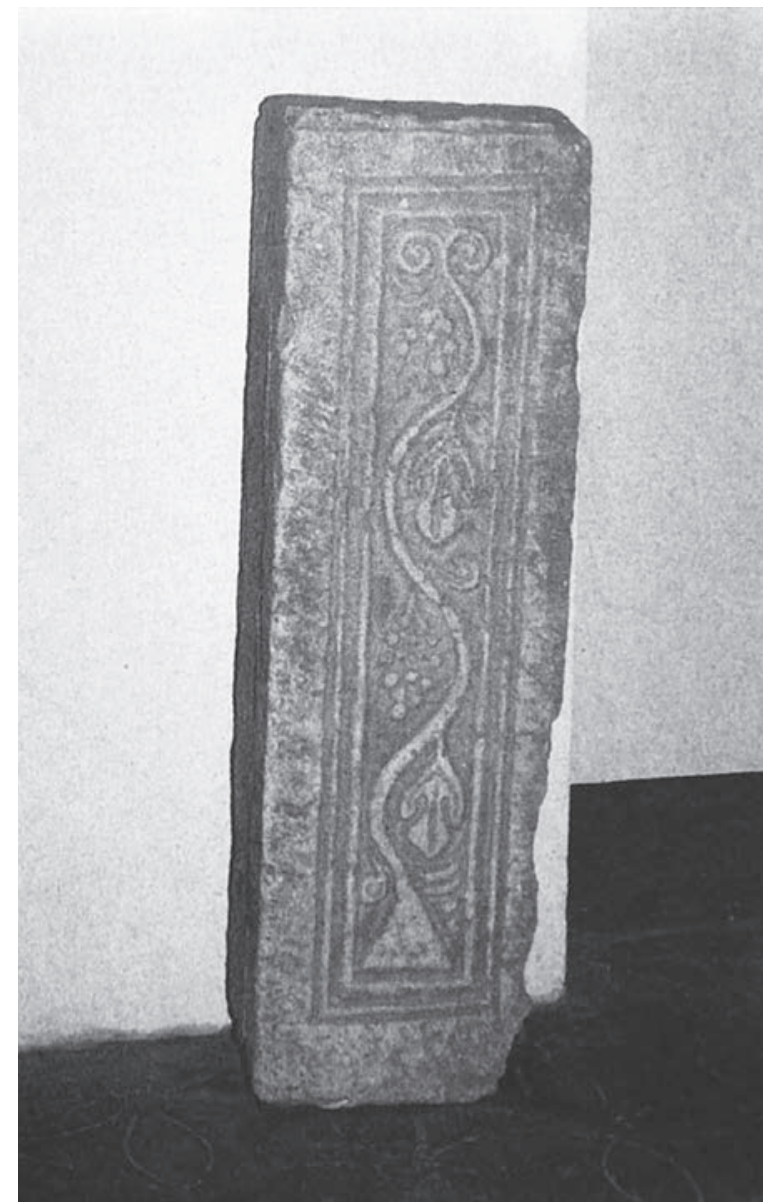

Fig. 7. Fossombrone, Museo Civico A. Vernarecci: pilastrino (da Guidobaldi, Guiglia Guidobaldi, Barsanti 1992).

tirrenica ${ }^{40}$. Analogamente, nel caso di Fossombrone si può ipotizzare una circolazione di maestranze o modelli lungo la direttrice della via Flaminia.

Un'origine greca è, invece, ipotizzabile per una parasta in marmo proconnesio del Museo Civico di Fano (Pesaro-Urbano), che mostra due pavoni ai lati di un cristogramma ${ }^{41}$.

\section{PUGLIA}

L'elevata concentrazione di sculture di importazione costantinopolitana lungo le coste pugliesi appare il riflesso della prosperità della regione tra il V e il VI secolo e del suo inserimento nelle reti di traffico che collegavano l'Adriatico e lo Ionio al più vasto ambito mediterraneo ${ }^{42}$.

\footnotetext{
${ }_{35}$ Su questi materiali, cfr. L. SERRA, Restauri e scoperte in S. Maria della Piazza di Ancona, in Bollettino d'Arte, 9, p. 97-121, in part. p. 102-103, 109. In S. Maria alla Piazza, l'esistenza di una fase giustinianea è testimonianza dal rifacimento dei rivestimenti pavimentali del IV/V secolo. Non è tuttavia chiaro se la ricostruzione dell'edificio sia avvenuta all'indomani della distruzione di Ancona da parte degli Ostrogoti nel 538 o del terremoto del 558 (BARSANTI, Mosaici... op. cit., p. 190-191).

${ }^{36}$ C. BARSANTI, La scultura paleocristiana e bizantina, in Scultura nelle Marche dalle origini all'età contemporanea, a cura di P. Zampetti, Firenze, 1993, p. 59-82, in part. 64; S. PIRANI, Considerazioni sui resti del sarcofago e sui monumenti epigrafici relativi ai SS. Pellegrino e Flaviano ad Ancona, in Atti del $V$ Congresso Nazionale di Archeologia Cristiana (Pesaro - Ancona, 19-23 settembre 1983), Firenze, 1985, p. 551-564, in part. 552-555.

37 BARSANTI, La scultura... op. cit., p. 64-65; PIRANI, Considerazioni... op. cit., p. 556-558.

$3^{8}$ BARSANTI, La scultura... op. cit., p. 65.

39 GUIDOBALDI, BARSANTI, GUIGLIA GUIDOBALDI, San Clemente... op. cit., p. 195-228.

${ }^{40}$ A GUIGLIA, Gli arredi liturgici di VI secolo, in Albenga. Un antico spazio cristiano. Chiesa e monastero di San Calocero al Monte. Un complesso archeologico dal I d.C. al XVI secolo, a cura di G. Spadea Noviero, Ph. Pergola, S. Roascio, Genova, 2010, p. 125-134.

${ }^{41}$ BARSANTI, La scultura... op. cit., p. 65.

${ }^{42}$ Sulla prosperità dell'Apulia tardoantica e sul suo ruolo di "ponte" tra l'Italia e l'Oriente, cf. G. VOLPE, San Giusto e l'Apulia nel contesto dell'Adriatico tardoantico, in L'archeologia dell'Adriatico dalla Preistoria al Medioevo, a cura di F. Lenzi (Atti del Convegno internazionale, Ravenna 7-8-9 giugno 2001), Firenze, 2001, p. 507-536, in part. 511-517.
} 


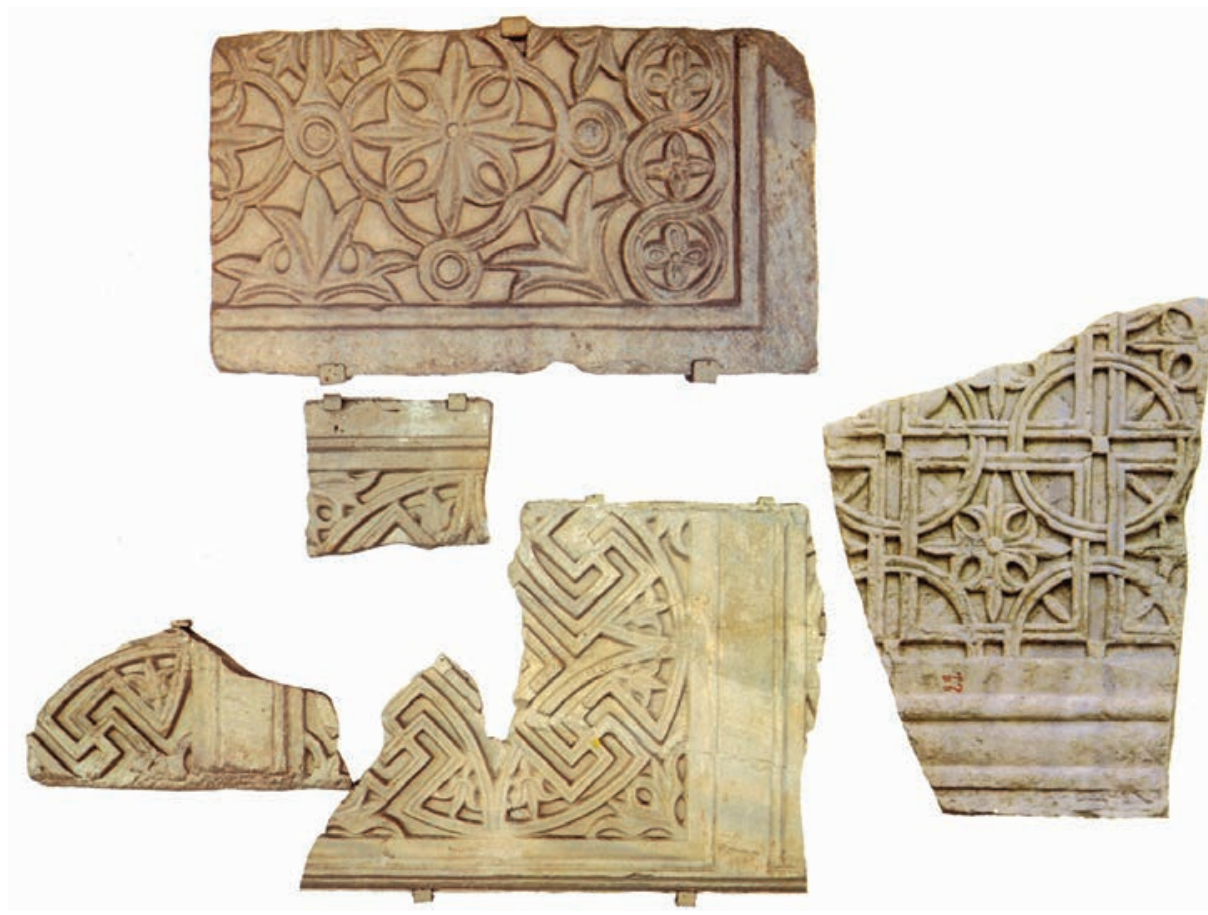

Fig. 8. Siponto, Museo Archeologico: pseudo-transenne (da Barsanti 1999). baccellate, con confronti ravennati oltre che in diverse località della Puglia ${ }^{47}$.

Alcune delle più interessanti testimonianze circa la diffusione di sculture costantinopolitane nell'area adriatica provengono da Siponto (Foggia), il principale porto dell'Apulia centro-settentrionale ${ }^{48}$. Capitelli "a lira" e capitelli compositi del tipo "ad acanto finemente dentellato" furono reimpiegati nella cattedrale medievale di S. Maria, assieme a basi di tipo attico e a fusti di colonna in cipollino dell'Eubea ${ }^{49}$. È probabile che questi elementi siano appartenuti alla vicina basilica episcopale paleocristiana, alla quale devono essere riferite anche le note pseudo-transenne del V/VI secolo con decorazione a meandri di svastiche semplici o complesse, disposti a formare cerchi o dodecagoni ${ }^{\circ}$ (fig. 8). Le lastre compongono un gruppo omogeneo, con confronti in Grecia, in Egitto e sul Monte Nebo in Giordania, e risultano chiaramente ispirate alle transenne dalla raffinata decorazione a giorno di Costan-

Anche a Bari, un centro di limitata importanza nel periodo tardoantico, numerosi spolia di V/VI secolo sono reimpiegati nelle chiese medievali della città ${ }^{43}$. Ė questo il caso della cattedrale romanica di S. Maria Assunta, che ha il proprio precedente in una basilica paleocristiana a tre navate, conclusa da un'abside e dotata di pastophoria ${ }^{44}$. In questo edificio erano originariamente collocate le base di colonna riutilizzate nel protiro dell'ingresso meridionale della cattedrale, che la decorazione a rombi concentrici con fiori quadripetali centrali permette di accostare ai 24 esemplari della basilica di Sant'Apollinare in Classe a Ravenna ${ }^{45}$. Anche altre chiese baresi (S. Nicola, S. Gregorio, S. Maria del Buonconsiglio, S. Pelagia/S. Anna) conservano marmi di importazione orientale $^{46}$, tra cui sono svariati esemplari di colonnine tortili e tinopoli (S. Sofia, SS. Sergio e Bacco), di Ravenna (basilica Ursiana, S. Vitale, S. Michele in Africisco, S. Apollinare Nuovo) e di località dell'Oriente mediterraneo quali Aizanoi (Turchia) e Gaza (Palestina) ${ }^{51}$.

La provenienza costantinopolitana delle transenne di Siponto è confermata dalla suggestiva testimonianza della Vita Laurenti episcopi sipontini, opera di ambito cassinese della fine dell'XI secolo, stando alla quale sarebbe stato addirittura l'imperatore Zenone (474-491) a invitare il consanguineo Lorenzo ad assumere la carica di vescovo di Siponto ${ }^{52}$. Una volta giunto a destinazione, Lorenzo si sarebbe rivolto all'augusto parente per chiedere l'invio di doctissimi artifices da impiegare nella costruzione e nella decorazione di edifici di culto, ottenendo anche un donativo di 150 libbre d'oro ${ }^{53}$.

\footnotetext{
$\overline{43}$ Su Bari tardoantica, si veda G. VOLPE, Contadini, pastori e mercanti nell'Apulia tardoantica, Bari, 1996, p. 137-141.

${ }^{44}$ G. BERTELLI, S. Maria que est episcopio. La cattedrale di Bari dalle origini al 1034, Bari, 1994.

${ }^{45}$ Gli spolia di S. Maria Assunta comprendono anche sette capitelli "a lira", due capitelli compositi del tipo "ad acanto finemente dentellato", un capitello "a calice" e due plutei frammentari: BERTELLI, Le diocesi... op. cit., p. 97-100, 102, 103-105

${ }^{46}$ In assenza di testimonianze archeologiche che consentano di attribuire un'origine paleocristiana a questi edifici, è probabile che gli elementi in questione provengano da strutture collocate nel territorio circostante la città: BERTELLI, Le diocesi... op. cit., p. 109-167.

${ }^{47}$ Sugli esemplari pugliesi, cfr. BERTELLI, Le diocesi... op. cit., p. 70; sul tipo, si veda R. FLAMINIO, Su un particolare tipo di colonna decorata di età paleocristiana, in Marmoribus vestita. Miscellanea in onore di Federico Guidobaldi, a cura di O. Brandt e Ph. Pergola (Studi di Antichità Cristiana 63), Città del Vaticano, 2011, p. 573-597.

${ }^{48}$ VOLPE, Contadini... op. cit., p. 121-124; sulle relazioni tra Siponto e il Mediterraneo orientale, cfr. D. NUZZO, L'epitaffio di un Siriano a Siponto e le relazioni della città con l'Oriente in età tardo antica, in Taras, 31, 2001, p. 191-208.

49 BERTELLI, Le diocesi... op. cit., p. 346-352.

${ }^{50}$ Agli esemplari di Siponto si devono aggiungere: una coppia di plutei portati a Benevento nell'alto Medioevo, in occasione dell'accorpamento delle due diocesi, i quattro di Monte Sant'Angelo, la lastra dell’abbazia di S. Maria di Pulsano e quella conservata al Nelson-Atkins Museum of Art di Kansas City (Stati Uniti), di sicura provenienza pugliese (C. BARSANTI, I plutei di Siponto, Monte Sant'Angelo e Benevento, in 1983-1993: dieci anni di archeologia cristiana in Italia, a cura di E. Russo (Atti del VII Congresso Nazionale di Archeologia Cristiana, Cassino, 20-24 settembre 1993), Cassino, 2003, p. 745-760; eadem, Una breve nota sui plutei di Siponto, Monte Sant'Angelo e Benevento, in Siponto antica, a cura di M. Mazzei, Foggia, 1999, p. 225-229).

${ }^{51}$ C. BARSANTI, Le transenne, in Santa Sofia di Costantinopoli. Santa Sofia di Costantinopoli. L'arredo marmoreo della Grande Chiesa giustinianea, a cura di A. Guiglia Guidobaldi e C. Barsanti (Studi di Antichità Cristiana 60), Città del Vaticano, 2004, p. 590-529, in part. p. 505-528.

${ }^{52}$ La Vita Laurenti episcopi sipontini ci è giunta in due recensiones, datate rispettivamente alla metà (Vita minor) e alla fine (Vita maior) dell’XI secolo (A. CAMPIONE, Lorenzo di Siponto: un vescovo del VI secolo tra agiografia e storia, in Vetera Christianorum, 41, 2005, p. 61-82; eadem, Storia e santità nelle due Vitae di Lorenzo di Siponto, in Vetera Christianorum, 29, 1992, p. 169-213).

53 La fonte presenta aspetti problematici: innanzitutto, la data dell'episcopato di Lorenzo va abbassata di circa tre decenni, dall'età di Zenone a quella di Giustiniano; in secondo luogo, la Vita Laurenti appare ispirata a quella dell'abate Desiderio di Montecassino (1058-1087), che inviò a Costantinopoli alcuni artigiani perché fossero istruiti nell'arte della lavorazione dei metalli, per poi reclutare maestranze bizantine disposte a insegnare la propria arte ai monaci
} 
Transenne analoghe a quelle sipontine provengono dal santuario micaelico di Monte Sant'Angelo sul Gargano, le cui origini sono fatte risalire proprio all'episcopato di Loren$\mathrm{zo}^{54}$. Fatto interessante, la tradizione agiografica attesta un precoce interesse bizantino per il santuario, che la giovane Artellaidis avrebbe visitato e omaggiato con un dono di "triginta aureos", dopo essersi rifugiata in Apulia presso lo zio Narsus (probabilmente Narsete) per sfuggire all'imperatore Giustiniano ${ }^{55}$.

Un altro centro in cui l'influsso dei modelli artistici metropolitani risulta particolarmente evidente è Canosa, dove la costruzione della cattedrale, ispirata alla basilica di S. Giovanni Evangelista a Efeso, e della chiesa di San Leucio, un edificio a pianta centrale a doppio tetraconco, va attribuita all'iniziativa del vescovo Sabino (in carica tra il 531 e il $542 / 552)$, che visitò ripetutamente la capitale d'Oriente ${ }^{56}$. È forse al ciborio o alla pergula di una di queste due basiliche che va riferito un capitello bizonale ad acanto finemente dentellato e arieti angolari, con confronti a Costantinopoli, Salonicco, Filippi, Cartagine (Damous-el-Karita) ea Ravenna (Sant'Apollinare in Classe) ${ }^{57}$.

Materiali di imitazione costantinopolitana sono stati recuperati nel corso degli scavi della cattedrale di Barletta ${ }^{58}$. Essi comprendono un pilastrino di recinzione presbiteriale, una colonnina frammentaria pertinente a una pergula o al sostegno di una mensa d'altare e il frammento di un parapetto di ambone a doppia rampa scalare59, tutti riferibili all'arredo di un edificio di culto il cui pastophorion "di tipo elladico" richiama soluzioni attestate nella Grecia continentale e insulare ${ }^{60}$.
Arredi di imitazione costantinopolitana sono presenti anche nella basilica di Canne della Battaglia (Barletta), dove si trovano tre capitelli-imposta ionici ${ }^{61}$. A Trani, la cattedrale di S. Maria e la sua cripta longitudinale, che occupano l'area di una basilica del V/VI secolo, reimpiegano diversi elementi di importazione, tra cui - oltre ad alcuni capitelli "ad acanto spinoso" e capitelli "ad acanto finemente dentellato" - è una colonnina tortile e baccellata ${ }^{62}$.

Particolarmente nutrito il numero degli spolia della cripta romanica della cattedrale di Otranto, preceduta anch'essa da una basilica paleocristiana. Oltre a materiali di epoca romana provenienti dall'anfiteatro di Lecce, la struttura conserva capitelli di diversa tipologia (corinzi, "a lira", "ad acanto spinoso", bizonali"), due pilastrini di recinzione presbiteriale e sei colonnine, il cui fusto si caratterizza per l'insolita decorazione a girali con mezze palmette e foglie trilobate ${ }^{63}$.

\section{EPIRO}

La carta di distribuzione della scultura costantinopolitana in Epiro dimostra come la circolazione di questi manufatti sia stata ostacolata dalla natura prevalentemente montuosa del territorio ${ }^{64}$. Non è un caso che nelle località dell'interno, l'uso del marmo sia limitata a elementi di piccole dimensioni, come le mense, i sostegni e le basi d'altare di Byllis ${ }^{65}$, la recinzione presbiteriale della basilica di Phoinike ${ }^{66}$ o quella della chiesa extra muros di Elbasan, dove plutei in marmo nero con venature grigie erano accostati a pilastrini in marmor Sagarium ${ }^{67}$.

(CAMPIONE, Storia... op. cit., p. 199-202). Tuttavia, i rapporti di Siponto con l'Oriente e la stessa presenza in città di sculture costantinopolitane danno credito alla veridicità del racconto della Vita Laurenti.

54 CAMPIONE, Storia..., p. 187-197.

${ }^{55}$ La vicenda è narrata nella Vita sanctae Artellaidis, redatta a Benevento nell'VIII secolo: G. BERTELLI, Pellegrinaggi femminili a Monte S. Angelo fra VI e VIII secolo, in Akten des XII. Internationalen Kongressus für Christliche Archäologie (Bonn, 22-28. September 1991), a cura di E. Dassmann e J. Engelmann (Studi di Antichità Cristiana 46), Città del Vaticano, p. 537-542, in part. p. 538-541.

${ }^{56}$ Sulla cattedrale di Canosa, G. BERTELLI, A.F. ATTOLICO, Analisi delle strutture architettoniche della Cattedrale di San Sabino a Canosa: primi dati, in Canosa. Ricerche storiche, decennio 1999-2009, a cura di L. Bertoldi Lenoci, Martina Franca, 2010, p. 724-758; sulla basilica di San Leucio, P. PENSABENE, A. D’ALESSIO, Il complesso di san Leucio alla luce dei nuovi scavi 2005-2006 a Canosa, in Canosa. Ricerche storiche 2007, a cura di L. Bertoldi Lenoci, Martina Franca, 2008, p. 105-142. Per un profilo biografico di Sabino, si veda Prosopographie de l'Italie chrétienne (314-604), a cura di Ch. Pietri e L. Pietri, Rome, 1999-2000, p. 1446-1452.

${ }^{57} \mathrm{Il}$ capitello fa parte di un gruppo di materiali di importazione comprendente anche alcuni pilastrini di recinzione presbiteriale e una lastra a modanature complesse con fiorone quadripetalo centrale (BERTELLI, Le diocesi... op. cit., p. 243, 255-256).

${ }^{58}$ La chiesa, individuata sotto le strutture della cattedrale romanica, ha restituito un mattone bollato con il monogramma di Sabino di Canosa, cui probabilmente si devono la costruzione dell'edificio e la promozione del vicus di Barletta al rango di sede episcopale: P. FAVIA, R. GIULIANI, Preesistenze sacre nel sottosuolo della cattedrale di Barletta, in Vetera Christianorum, 34, 1997, p. 329-365, in part. p. 331-347.

${ }^{59}$ R. GIULIANI, Gli arredi plastici della basilica paleocristiana di Barletta, in Vetera Christianorum, 36, 1998, in part. p. $305-306$ e 310.

${ }^{60}$ P. FAVIA, R. GIULIANI, Preesistenze... op. cit., p. 341-342.

${ }^{61}$ BERTELLI, Le diocesi... op. cit., p. 208, 211-212, 221-222, 223-224, 224-225

${ }^{62}$ BERTELLI, Le diocesi... op. cit., p. 356-366; P. PENSABENE, Capitelli bizantini e bizantineggianti della cripta del duomo di Trani, in Bisanzio e l'Occidente: arte, archeologia. Studi in onore di Fernanda de' Maffei, a cura di C. Barsanti, Roma, 1996, p. 375-408.

${ }^{63}$ M. FALLA CASTELFRANCHI, Sul Bosforo d'Occidente: la cultura artistica di Otranto in epoca tardoantica e medioevale, in Otranto nel Medioevo tra Bisanzio e l'Occidente, a cura di H. Houben, Galatina, 1997, p. 281-324, in part. p. 283-286; P. VERGARA, Elementi architettonici tardoantichi e medioevali nella cripta della Cattedrale di Otranto, in Rivista dell'Istituto Nazionale di Archeologia e Storia dell'Arte, 4, 1981, p. 71-103, in part. p. 75-77, 80-82 e 99-102; sulle colonnine, variamente datate tra il VI e l'XI secolo, cfr. FLAMINIO, Su un particolare tipo... op. cit., p. 596-597.

${ }^{64}$ W. BOWDEN, Epirus Vetus. The Archaeology of a Late Antique Province, London 2003, p. 114-121. Esemplificativo il caso di Byllis, dove cave di calcare sono state individuate all'esterno come all'interno della cerchia muraria giustinianea, alcune delle quali collocate presso le grandi basiliche paleocristiane della città (T. KOŽELJ, M. WURCH KOŽELJ, Grey Limestone Quarries of Byllis (Albania), in Interdisciplinary Studies on Ancient Stone. Proceedings of the IX ASMOSIA Conference (Tarragona 2009), p. 624-627), a cura di A. Gutiérrez Garcia-Moreno, P. Lapuente, I. Rodà de Llanza, Tarragona, 2012, p. 619-627.

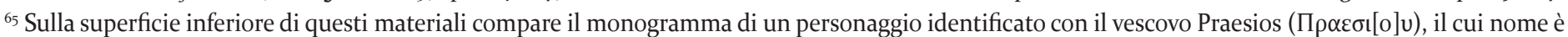
presente anche nelle iscrizioni musive del V/VI secolo delle basiliche C e D: P. CHEVALIER, Les autels paléochrétiens des provinces d'Epirus Vetus, Epirus Nova et de Praevalis, in Hortus Artium Medievalium, 11, 2005, p. 65-80, in part. p. 73-75.

${ }^{66}$ Dalla basilica di Phoinike provengono anche un capitello a stampella e alcuni frammenti di colonna tortile, tutti in marmo: E. HOBDARI, M. PODINI, Edilizia ecclesiastica e reimpiego nelle chiese di V-VI secolo e XI-XII secolo nel territorio di Phoinike e Butrinto, in Ocnus, 16, 2008, p. 147-172, in part. p. 162.

${ }^{67}$ E. HOBDARI, Karakteristika të skulpturës paleokristiane në Shqipëri, in Iliria, 35, 2011, p. 351-362, in part. p. $342-345$ (con riassunto italiano). 
Le importazioni risultano assai più numerose nelle città della costa e in quelle collocate lungo il tracciato della via Egnatia, di cui Durazzo, oltre a essere uno dei porti principali dell'intero Adriatico, era il terminale occidentale. Qui, l'imperatore Anastasio (491-518), che della città era originario, patrocinò la costruzione del Foro Circolare ${ }^{68}$ (fig. 9), la cui piazza è delimitata da colonne in granito della Troade, abbinate a basi e capitelli corinzi in marmo del Proconneso $^{69}$.

A breve distanza da Durazzo, anche l'arredo della basilica giustinianea di Arapaj è prevalentemente in marmo proconnesio $^{70}$. A Saranda (l'antica Onchesmos), scalo di primaria importanza lungo le rotte che collegavano l'Adriatico allo Ionio ${ }^{71}$, lo scavo di Rruga Skenderbeu ha riportato alla luce i resti di una basilica del V/VI secolo, installata all'interno di una sinagoga più antica di circa un seco$\mathrm{lo}^{72}$. L'arredo dell'edificio comprendeva una recinzione presbiteriale in marmo, completa di cimase decorate con croci semplici e su disco ${ }^{73}$, una mensa d'altare iscritta e diversi capitelli bizonali ${ }^{74}$.

A est di Saranda, sulla collina che domina la città e il golfo sottostanti, sorge la basilica dei Quaranta Martiri, un edificio del V/VI secolo sviluppato su due livelli: una chiesa superiore, dalla pianta mononave a eptaconco preceduta da un nartece, e una cripta anulare, sulla quale si aprono dieci cappelle ${ }^{75}$. Alla complessità architettonica della basilica fa però riscontro la semplicità dell'arredo liturgico, interamente realizzato in calcare locale ${ }^{76}$.

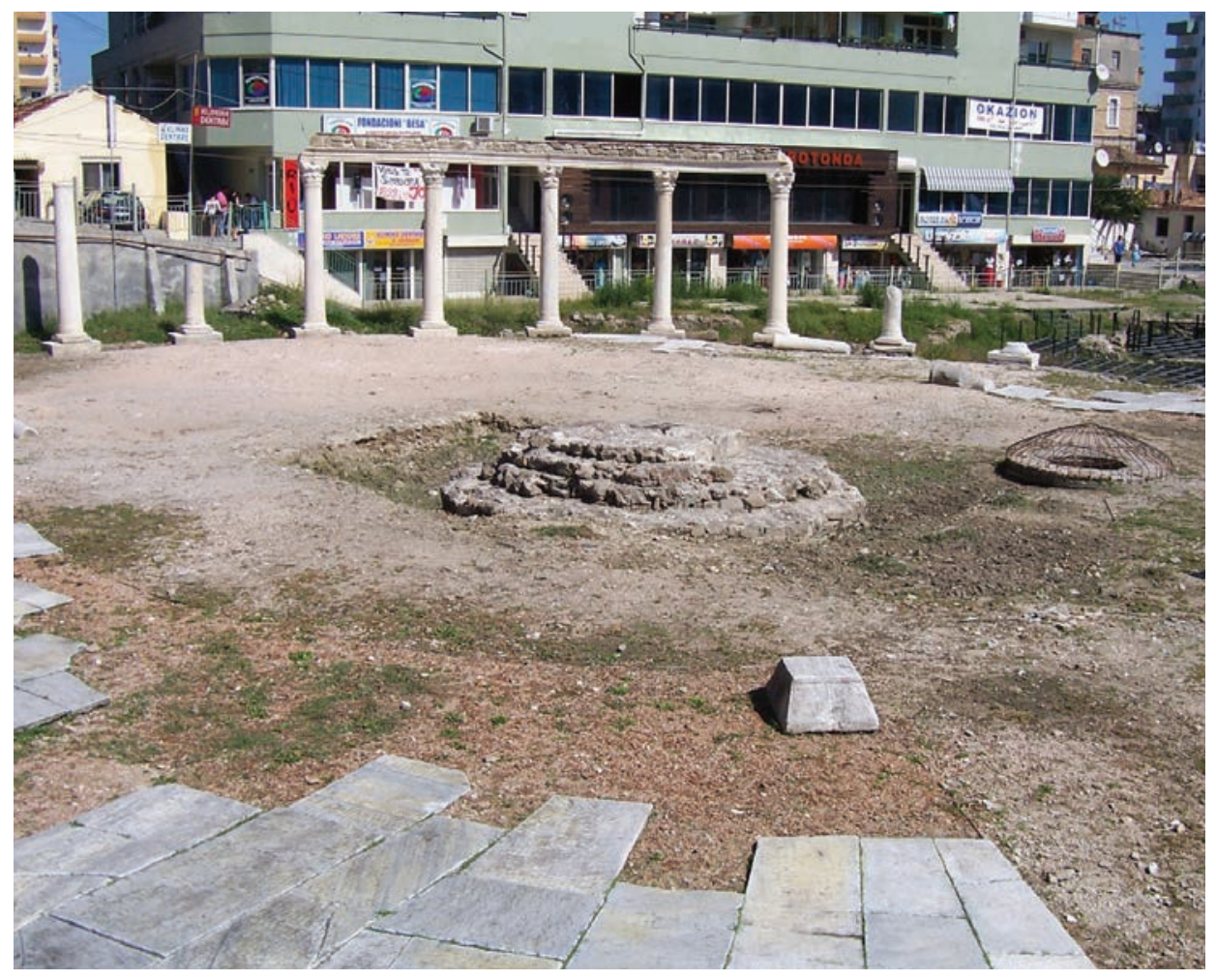

Fig. 9. Durazzo, Foro Circolare (foto autore).

L'utilizzo del calcare caratterizza anche la scultura di Butrinto, dove gli elementi in marmo compongono un insieme piuttosto ridotto, di cui fanno parte - oltre alla recinzione presbiteriale e alle imposte della Grande Basilica (secondo venticinquennio del VI secolo) ${ }^{77}$ - anche i capitelli a stampella, una colonnina di finestra e una transenna con decorazione a giorno dalla basilica dell'Acropoli ${ }^{7}$. Un caso particolare è quello del battistero, i cui capitelli-imposta ionici, collocati su colonne in granito della Troade, furono scolpiti utilizzando marmi di recupero ${ }^{79}$.

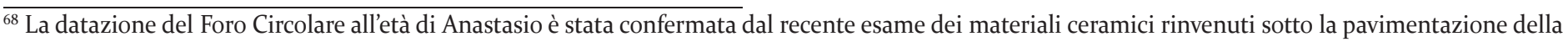
piazza: A. HOTI, J. WILKES, E. METALLA, B. SKHODRA, The Early Byzantine Circular Forum in Dyrrachium (Durrës, Albania) in 2002 and $2004-2005:$ Recent Recording and Excavation, in The Annual of the British School at Athens, 103, 2008, p. 367-397, in part. p. 394-395. Sull'attività edilizia di Anastasio a Durazzo, cui le fonti attribuiscono anche la costruzione di un circo e di una cerchia muraria, cfr. F.K. HAREER, Anastasius I. Politics and Empire in the Late Roman World (ARCA. Classical and Medieval Texts. Papers and Monographs 46), Cambridge, 2006, p. 242-244.

${ }^{69}$ La provenienza di questi elementi dalle cave del Proconneso è certificata dalla presenza di masons' marks in lettere greche (ПAT e EY), che compaiono su alcune basi, capitelli e fusti di colonna: P. PENSABENE, Inscribed Architectural Elements from the Prokonnesos in Durazzo, Tartous, Cilician Aphrodisias, and Caesarea, in ASMOSIA V. Interdisciplinary Studies on Ancient Stones, a cura di J.J. Herrmann, N. Herz, R. Newman, London, 2002, p. 328-334, in part. p. 329-330). Presso il museo archeologico di Durazzo si conservano numerosi manufatti in marmo e in pietra locale (A. HOTI, Plastike arkitektonike decorative paleokristiane nga qyteti i Durrësit, in Iliria, 1-2, 1997, p. 325-341), tra i quali spicca un capitello bizonale (S. ANAMALI, Kapitell, in Albanien. Schätze aus dem Land der Skipetaren (Catalogo della mostra, Hildesheim 1988), Mainz am Rhein, 1988, p. 446).

$7^{\circ}$ H. HIDRI, S. HIDRI, Die frühchristliche Basilika in Arapaj/Durrës (Albanien), Wien 2012, p. 31-32.

${ }^{71}$ R. HODGES, Sarandë, ancient Onchesmos, and the 'corrupting sea', in New Directions in Albanian Archaeology. Studies presented to Muzafer Korkuti, a cura di L. Bejko e R. Hodges (International Centre for Albanian Archaeology Monograph Series 1), Tirana, 2006, p. 223-241, in part. p. $223-229$.

${ }^{72}$ E. NALLBANI ET ALII, La synagogue antique d'Anchiasmos, en Albanie, in L'archéologie du judaïsme en France et en Europe, a cura di P. Salmona e L. Sigal, Paris, 2011, p. 63-73

${ }^{73}$ K. LAKO, Basilika paleokristiane e Onhezmit, in Iliria 21, 1991, p. 123-186, in part. p. 140. Per una serie di confronti albanesi, cfr. E. HOBDARI, Dorëmbështetësit në instalimet e bazilikave paleokristiane, in Candavia, 2, 2005, p. 131-135.

${ }_{74}^{74}$ LAKO, Basilika... op. cit., p. 134-136; HOBDARI, Karakteristika... op. cit., p. 344-346.

75 J. MITCHELL, The Archaeology of Pilgrimage in Late Antique Albania: the Basilica of the Forty Martyrs, in Recent Research on the Late Antique Countryside, a cura di W. Bowden, L. Lavan, C. Machado (Late Antique Archaeology 2), Leiden-Boston, p. 86-105.

${ }^{76}$ HOBDARI, PODINI, Edilizia..., op. cit., p. 153.

77 W. BOWDEN, J. MITCHELL, The Christian Topography of Butrint, in Byzantine Butrint. Excavations and Surveys 1994-1999, a cura di R. Hodges, W. Bowden, K. Lako, Oxford, 2004, p. 104-125, in part. p. 106-111. L'arredo architettonico della Grande Basilica comprendeva anche materiali di reimpiego: HOBDARI, PODINI, Edilizia..., op. cit., p. 151-152.

${ }^{78}$ BOWDEN, MITCHELL, The Christian Topography..., op. cit., p. 111.

79 W. BOWDEN, L. PËRZHITA, The Baptistery, in Byzantine Butrint. Excavations and Surveys 1994-1999, Oxford, 2004, p. 176-201, in part. p. 185-190. I capitelli del battistero di Butrinto possono essere confrontati con le imposte rinvenute ad Hadrianopolis, a una sessantina di chilometri da Butrinto. Scolpite in
} 


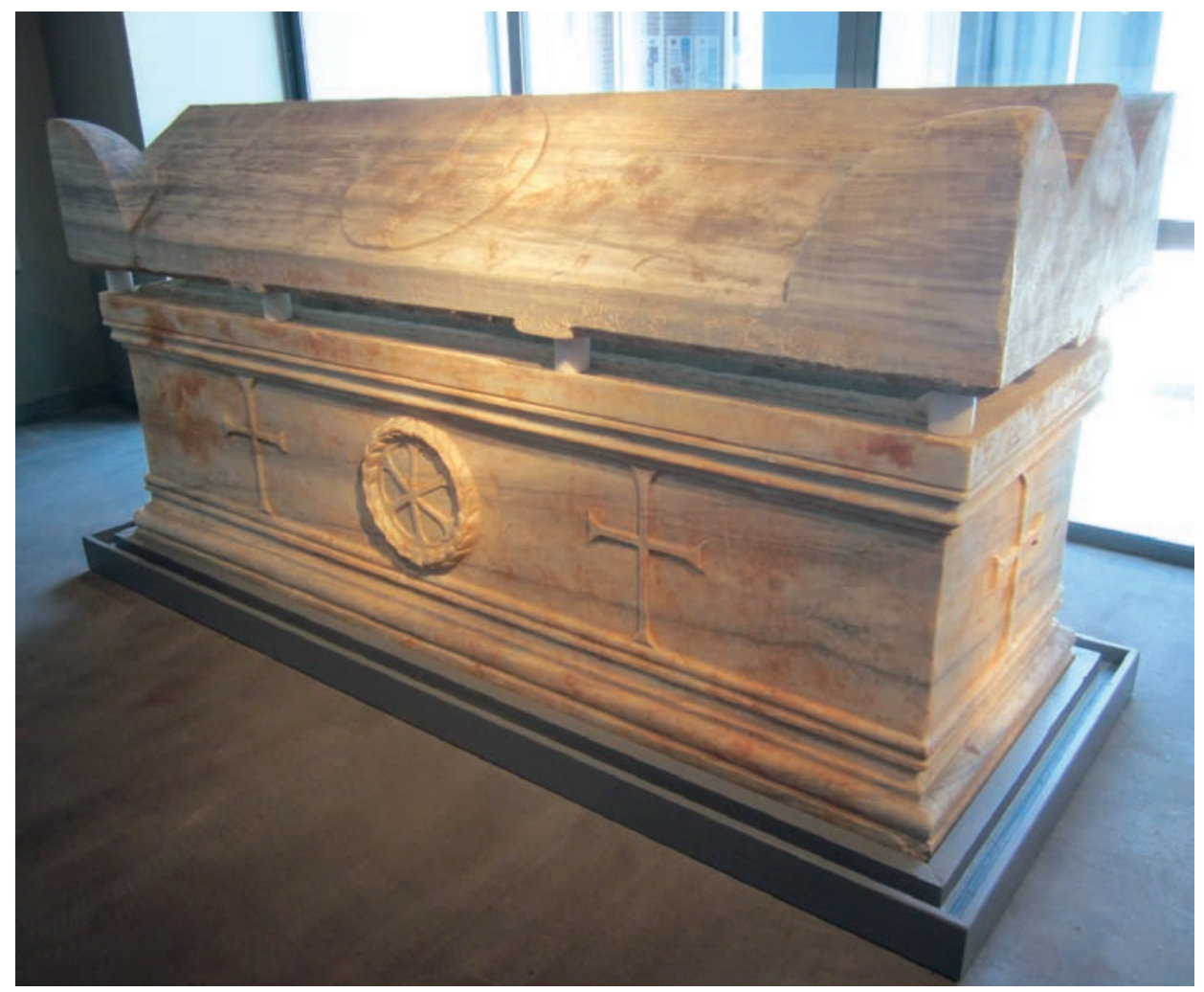

Fig. 10. Nikopolis, Museo Archeologico: sarcofago dalla basilica $\Delta$ (foto autore).

dal braccio settentrionale di quest'ultima proviene un monumentale sarcofago a cassapanca con decorazione simbolica e coperchio a doppio spiovente e acroteri angolari (fig. 10), che, utilizzato per la sepoltura di un vescovo o di un martire, offre una rara testimonianza della circolazione oltre l'ambito costantinopolitano di questi prestigiosi manufatti ${ }^{83}$.

\section{CONCLUSIONI}

Come in altre regioni del Mediterraneo tardoantico, anche nell'Adriatico la circolazione della scultura costantinopolitana appare legata al potere imperiale e ai suoi rappresentanti. A Ravenna, le imposte della basilica di S. Giovanni Evangelista, fondata da Galla Placidia tra il 424 e il 434, e i ventiquattro fusti di colonna, capitelli e imposte della basilica Apostolorum, anch'essa di probabile fondazione imperiale, costituiscono il primo esempio dell'importazione di setti omogenei di elementi architettonici in marmo del Proconneso. Un uso, questo, destinato a diventare pratica corrente nel prosieguo del V e nel corso del VI secolo,

Un analogo accostamento di materiali in calcare, spolia e marmi di importazione contraddistingue anche l'arredo delle basiliche paleocristiane di Nikopolis, capitale della provincia tardoantica dell'Epirus vetus ${ }^{80}$. Il rinvenimento negli edifici di culto della città di capitelli “a lira”, di capitelli-imposta ionici e di fusti di colonna in marmo proconnesio rimanda a Costantinopoli, dove furono realizzate anche le transenne a giorno della basilica $\mathrm{B}^{81}$, che servirono da modello per quelle in pietra locale della basilica $\Delta^{{ }^{2}}$. Proprio in coincidenza con il periodo di massima attività edilizia nella storia tardoantica della città, dove è stato calcolato che, tra gli episcopati di Giovanni I (477-494) e di Pietro III (570578), siano giunte all'incirca 1.556 tonnellate di marmo ${ }^{84}$.

Un simile afflusso di materiali ha spinto a supporre che a Ravenna fosse presente un deposito centrale dei marmi di importazione che, modellato sulla cosiddetta statio marmorum della Roma imperiale, avrebbe provveduto all'immagaz-

pietra locale, le imposte mostrano una banale decorazione con croci patenti e lemnischi, senza che nulla suggerisca il benché minimo rimando ad ancestrali simbologie pagane (pace G. MONTALI, Imposte di pilastro, in Hadrianopolis II. Ricerche e indagini archeologiche 2005-2010, a cura di R. Perna e D. Çondi (Bibliotheca Archaeologica 29), Bari, 2012, p. 218-221.

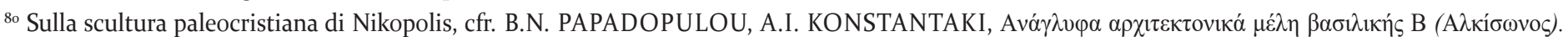

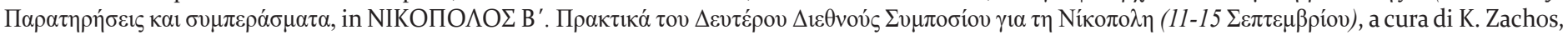
Preveza, 2007, p. 637-657, e E. CHALKIA, La scultura paleocristiana di Nikopolis. Alcune considerazioni, in Akten des XIV. Internationalen Kongresses für Christliche Archäologie. Frühes Christentum zwischen Rom und Konstantinopel (Wien 19.-26. 9. 1999), a cura di Ph. Harreiter, R. Pillinger, A. Pülz (Studi di Antichità Cristiana 62), Città del Vaticano, 2006, p. 281-289.

${ }^{81}$ CHALKIA, La scultura... op. cit., p. 285-287.

${ }^{82}$ PAPADOPULOU, KONSTANTAKI, Avóx $\lambda v \varphi \alpha \ldots$ op. cit., p. 648; CHALKIA, La scultura... op. cit., p. 282-283. Nella Grecia nord-occidentale, transenne

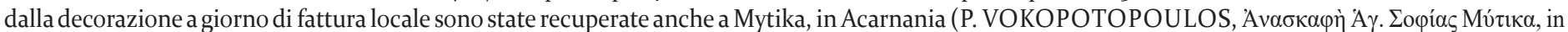

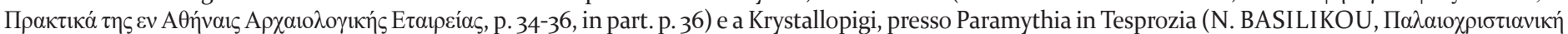

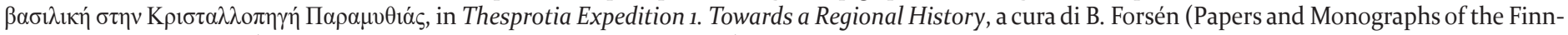
ish Institute at Athens 15) Helsinki, 2009, p. 197-206, in part. p. 203-204).

${ }_{3}^{8}$ Delle dimensioni di 2,67 m per o,88 m, il sarcofago è decorato sui lati lungo secondo lo schema ternario del chrismon entro serto di alloro tra due croci patenti. Croci patenti compaiono anche sui lati brevi della cassa, mentre sul coperchio, a doppio spiovente e acroteri angolari, sono due croci su disco (E. CHALKIA, Un sarcofago costantinopolitano a Nicopoli, in Rivista di Archeologia Cristiana, 80, 2004, p. 211-232). Il sarcofago trova confronti a Costantinopoli (R. FLAMINIO, I sarcofagi bizantini del Museo di Santa Sofia a Istanbul, in Vie per Bisanzio. VII Congresso Nazionale dell'Associazione Italiana di Studi Bizantini (Venezia, 25-28 novembre 2009), a cura di A. Rigo, A. Babuin, M. Trizio, Bari, 2011, p. 455-476), a Doljani in Montenegro (I. NIKOLAJEVIĆ STOJKOVIĆ, La décoration architecturale des églises découvertes à Doljani - Monténégro, in Atti del VI Congresso Internazionale di Archeologia Cristiana (Ravenna, 23-30 settembre 1962) (Studi di Antichità Cristiana 26), Città del Vaticano, 1965, p. 457-470, in part. p. 463-468) e a Naxos nelle Cicladi (M. LAMBERTZ, Ein frühchristlicher Sarkophag aus Naxos, in Mitteilungen zur Spätantiken Archäologie und Byzantinischen Kunstgeschichte 5, 2007, p. 21-33). Inoltre, un coperchio a doppio spiovente e acroteri angolari, decorato sui due lati da una croce patente, è stato rinvenuto durante lo scavo della basilica di

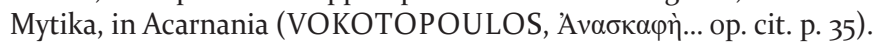

${ }^{84}$ Si tratta di una stima prudente, che considera solo gli elementi architettonici (fusti di colonna, capitelli, basi e blocchi di imposta), non i marmi importati sotto forma di arredi liturgici, sarcofagi e lastre di rivestimento: J.G. HARPER, The Provisioning of Marble for Sixth Century Churches of Ravenna: a Reconstructive Analysis, in Pratum Romanum. Richard Krautheimer zum 10o. Geburstag, a cura di R.L. Colella, M.J. Gill, L.A. Jenkens, P. Lamers, Wiesbaden, 1997, p. 131-148, in part. p. 145-147. 
zinamento e alla ridistribuzione di questi materiali verso $\mathrm{i}$ cantieri attivi in città ealtrove ${ }^{85}$. Tuttavia, non solo l'esistenza di un simile sistema centralizzato appare dubbia già per il periodo romano ${ }^{86}$, ma essa risulta tanto più inverosimile nel contesto del Mediterraneo tardoantico, caratterizzato da frammentazione politica, da una netta flessione dell'attività edilizia e da un concomitante calo del consumo del marmo e della pietra in genere ${ }^{87}$. Ė probabile che la circolazione della scultura costantinopolitana nell'Adriatico del V e VI secolo sia da riferirsi all'iniziativa delle singole committenze, che si approvvigionavano di tali materiali direttamente nella capitale imperiale, senza passare per il tramite di Ravenna ${ }^{88}$.

Se a Durazzo la decorazione del Foro Circolare va ricondotta all'imperatore Anastasio, altrove l'importazione di marmi avvenne comunque nell'ambito di progetti ufficiali. Un caso emblematico è rappresentato da Ancona, la cui importanza si accrebbe notevolmente nel corso dello scontro con gli Ostrogoti, fino a soppiantare la vicina Osimo, di cui era stata fino ad allora il sobborgo portuale ${ }^{89}$. Il restauro degli edifici di culto cittadini e la presenza al loro interno di sculture costantinopolitane fa intravedere l'attenzione tributata dalle gerarchie bizantine alla riorganizzazione delle strutture ecclesiastiche (anche di quelle materiali) del Piceno. Un altro esempio particolarmente eloquente è quello di Pesaro, dove nel VI secolo la stesura del tappeto musivo della cattedrale fu oggetto di un esteso rifacimento, che un'iscrizione musiva nella navata centrale attribuisce alla committenza di vir gloriosus magister militum et exconsul provincia Mysiae natus Giovanni, comandante delle truppe imperiali nel Piceno e in Apulia durante la guerra gotica, e a un certo Narses, senza dubbio il generalissimo Narsete ${ }^{90}$.
L'analisi della documentazione storica e archeologica permette, inoltre, di apprezzare il ruolo dei vescovi nella circolazione nell'area adriatica dei manufatti marmorei e dei modelli artistici costantinopolitani. è noto come la costruzione e la decorazione degli edifici di culto fosse una componente fondamentale delle strategie di affermazione e di autorappresentazione dell'autorità episcopale ${ }^{91}$, e le fonti attestano il coinvolgimento dei vescovi nella produzione e nell'approvvigionamento dei materiali da costruzione ${ }^{92}$. Per quel che concerne il marmo, si può citare la testimonianza del Liber Pontificalis Ecclesiae Ravennatis, in cui Agnello ricorda la sostituzione delle antiche colonne in legno di noce della basilica di Sant'Andrea di Ravenna con fusti in marmo proconnesio, forniti dall'arcivescovo Massimiano ${ }^{93}$. Salito sulla cattedra episcopale ravennate per volontà dello stesso Giustiniano, Massimiano mantenne sempre un rapporto privilegiato con Costantinopoli, come pare essere stato il caso anche di Lorenzo di Siponto e di Sabino di Canosa94. Per questa ragione, similmente a quanto proposto per Roma, dove la presenza di arredi liturgici in marmo del Proconneso coincide cronologicamente con il reciproco scambio di epistole e doni diplomatici tra Giustiniano e i papi Ormisda (514-523), Giovanni I (523-535) e Giovanni II (533-535)95, anche nell'Adriatico del Ve VI secolo la circolazione di sculture, manufatti artistici e di maestranze costantinopolitane deve avere rappresentato il corollario di relazioni di altra natura ${ }^{96}$.

Uomini, idee e oggetti dovevano, dunque, muoversi lungo la fitta trama di rotte marittime che univa da secoli le regioni adriatiche al Mediterraneo orientale, intersecandosi a direttrici terrestri quali la via Egnatia. Il tutto contribuì alla formazione di una koiné artistica adriatica che aveva

\footnotetext{
${ }_{85}$ HARPER, The Provisioning... op. cit., p. 134-140.

${ }^{86}$ RUSSELL, The Economics... op. cit., 232-234.

${ }^{87}$ La proposta di HARPER, The Provisioning... op. cit., p. 134, di individuare il sito della statio marmorum ravennate presso la chiesa di S. Giovanni in Marmorata, collocata a nord di Ravenna, in prossimità della Porta Guarcinorum, appare piuttosto fragile: nel periodo medievale e moderno, l'area della statio marmorum di Roma, ai piedi dell'Aventino, era effettivamente nota come "Marmorata", ma nulla esclude che nel contesto ravennate il toponimo indicasse una zona caratterizzata dalla presenza di rovine e macerie. Allo stesso modo, la notizia tramandata da Cassiodoro (Variae III, 19), secondo cui Teoderico avrebbe concesso al marmorarius Daniele il controllo sulla fornitura di sarcofagi alla corte di Ravenna, non può essere utilizzata per sostenere l'esistenza in città di un centro organizzato di raccolta e ridistribuzione dei marmi importati o quella di un funzionario preposto alla loro ridistribuzione. Del resto, nella lettera Cassiodoro fa riferimento a una molteplicità di scultori e venditori di sarcofagi, smentendo dunque la possibilità che il marmorarius abbia esercitato un monopolio su tale attività: Y.A. MARANO, Cassiodorus on Marble, in ASMOSIA X. Proceedings of the Tenth Interdisciplinary Studies on Ancient Stone, a cura di P. Pensabene e E. Gasparini, Roma, 2015, p. 997-1003, in part. p. 999-1001.

${ }^{88}$ Y.A. MARANO, Marmo e committenze nell'Adriatico tardoantico (V-VI secolo d.C.), in Economia e territorio nell'Adriatico centrale tra tarda Antichità e alto Medioevo (IV-VIII secolo), a cura di E. Cirelli e E. Giorgi (Atti del convegno di studio, Ravenna, 28 febbraio - 1 marzo 2014), Bologna, in corso di stampa. ${ }^{89}$ Sulla crescita di Ancona durante l'età bizantina, cfr. E. ZANINI, Le Italie bizantine. Territorio, insediamenti ed economia nella provincia bizantina d'Italia (VI-VIII secolo), Bari, 1998, p. 150-153.

${ }^{9}$ Sul mosaico e l'iscrizione, si veda R. FARIOLI CAMPANATI, I mosaici pavimentali della seconda fase della cattedrale di Pesaro, in Picus. Studi e ricerche sulle Marche nell'Antichità, 18, 1998, p. 7-29. Le fonti ricordano la partecipazione di Giovanni alle operazioni svolte dai Bizantini nel Piceno tra il 548 e il 551, quando egli ebbe un ruolo attivo nell'assedio di Ancona e nella conquista di Senigallia (Prosopographie de l'Italie chrétienne (314-604), a cura di Ch. Pietri e L. Pietri, Rome, 1999-2000, p. 1083-1084).

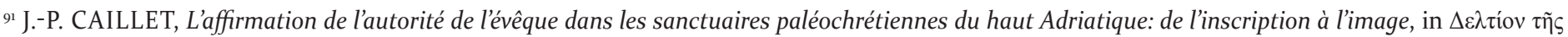

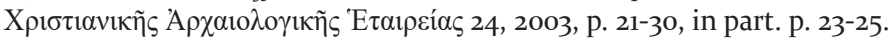

${ }_{92} \mathrm{G}$. VOLPE, Il ruolo dei vescovi nei processi di trasformazione del paesaggio urbano e rurale, in Archeologia e società tra tardo antico e alto medioevo, a cura di G.P. Brogiolo e A. Chavarría Arnau (Documenti di Archeologia 44), Mantova, 2007, p. 85-106, in part. p. 93-94. Una testimonianza significativa in proposito è offerta Liber Pontificalis Ecclesiae Ravennatis, in cui Agnello afferma che l'archiergatus della Chiesa di Ravenna si sarebbe rivolto all'arcivescovo Massimiano lamentando la penuria di "caementum et latercula ... lapides", spingendo il presule a intervenire e a fornire nottetempo quanto necessario al prosieguo dei lavori, "omnia paramenta, calces et latercula, petras et bisales, lapides et ligna, columnas et lastas, harenas et sabulos".

${ }_{93}$ Agnelli, Liber pontificalis ecclesiae ravennatis 76, ed. d. Mauskopf, Corpus Christianorum Series Latina. Continuatio Medievalis, 199, Turnhout, 2006, p. 244: [Maximianus] Ecclesiam uero beati Andreae apostoli hic Rauennae cum omni diligentia, non longe a regione Herculana, columnas marmoreas suffulsit, ablatas que uetustas ligneas de nucibus proconnisas decorauit.

94 Per tutta la tarda Antichità, i vescovi dell'Italia meridionale svolsero il ruolo di intermediari privilegiati della Chiesa di Roma presso il mondo bizantino (G. OTRANTO, Note sull'Italia meridionale paleocristiana nei rapporti con il mondo bizantino, in Augustinianum, 34, 1995, p. 859-884, in part. p. 864).

${ }_{95}$ A. GUIGLIA GUIDOBALDI, La scultura di arredo liturgico nelle chiese di Roma: il momento bizantino, in Ecclesiae urbis. Atti del Congresso internazionale di studi sulle Chiese di Roma (IV-X secolo) (Roma, 4-10 settembre 2000) (Studi di Antichità Cristiana 5), Città del Vaticano, 2002, p. 1479-1524, in part. p. 1490.
} 
nell'apertura verso l'Oriente una delle sue principali caratteristiche, con evidenti riscontri anche nell'ambito dell'architettura religiosa ${ }^{97}$ e della produzione musiva ${ }^{98}$. La diffusione lungo l'arco costiero adriatico dei capitelli-imposta ionici, scarsamente attestati al di fuori dell'Egeo e del Mediterraneo orientale, è sintomatica di un'unità destinata a sopravvivere anche all'esaurimento delle importazioni da Costantinopoli: alla fine del VI secolo i sarcofagi in calcare prodotti nelle cave dell'isola di Brač (in Dalmazia) e diffusi su ambo le sponde dell'Adriatico prenderanno il posto delle più raffinate arche costantinopolitane in marmo proconnesio, a testimonianza del restringimento degli orizzonti culturali della regione, ma anche della sua sostanziale omogeneità culturale ${ }^{99}$.

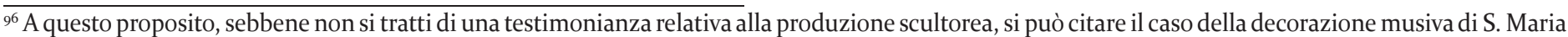
Formosa a Pola, di cui resta oggi solo un frammento raffigurante Cristo tra due apostoli o una scena di traditio legis, che i caratteri stilistici avvicinano ai mosaici dell'ambone della basilica B di Nikopolis e a opere di ambito orientale, come i mosaici della chiesa di Hosios David a Salonicco e della Panagia Kanakariá di Lythrankomi, a Cipro (A. GUIGLIA GUIDOBALDI, Il mosaico dell'ambone della basilica B di Nikopolis, in Nikopolis I. Proceedings of the International Symposium on Nikopolis. Preveza, 23-29 September 1984, a cura di E. Chrysos, Preveza 1987, p. 279-293). La grande raffinatezza tecnica e le piccole tessere che segnano i trapassi chiaroscurali nei volti orientano verso maestranze originarie dell'Oriente mediterraneo, come suggerito anche da un'iscrizione greca posta originariamente lungo il catino absidale e oggi scomparsa (S. Tavano, La restaurazione giustinianea in Africa e nell'arco Altoadriatico, in Aquileia e l’Africa (Antichità Altoadriatiche 5), Udine 1974, p. 251-283, in part. p. 252.

${ }_{97}$ A questo proposito, si possono citare i paralleli tra la basilica paleocristiana di S. Lorenzo ad Ancona, la basilica meridionale nel complesso episcopale di Salona, la chiesa di Oborci in Bosnia Erzegovina e una delle chiese dell'Hemmaberg in Austria (PANI ERMINI, La chiesa... op. cit.), così come tra il battistero di Butrinto e quelli di San Giusto (Lucera) e Nocera Inferiore (BOWDEN, PË̈RZHITA, The Baptistery... op. cit., p. 199). Per quel che concerne invece l'organizzazione degli spazi liturgici, si può sottolineare come nell'area adriatica risultino particolarmente diffuse le recinzioni presbiteriali "a $\Pi$ capovolto", una soluzione di origine costantinopolitana attestata anche nei Balcani (G. CUSCITO, L'arredo liturgico delle basiliche paleocristiane della Venetia orientale, in Hortus Artium Medievalium, 5, 1999, p. 87-104).

${ }_{98}^{8}$ Sulla diffusione di motivi di origine orientale e africana nella produzione musiva adriatica, cfr. R. FARIOLI CAMPANATI, Botteghe ravennati tra Oriente e Occidente, in Ravenna da capitale imperiale a capitale esarcale, Atti del XVII Congresso internazionale di studio sull'alto medioevo (Ravenna, 6-12 giugno 2004), Spoleto, 2005, p. 361-381, in part. p. 374-380.

${ }_{99}$ R. FARIOLI CAMPANATI, La scultura architettonica e di arredo liturgico a Ravenna alla fine della tarda Antichità: $i$ rapporti con Costantinopoli, in Storia di Ravenna dall'età bizantina all'età ottoniana, II.1. Territorio, economia e società, a cura di A. Carile, Venezia, 1991, pp. 249-267, in part. p. 264, sulla diffusione dei sarcofagi in pietra di Brač, C. D’ANGELA, Produzione e commercio di sarcofagi tra le due sponde adriatiche nel VI secolo, in La cristianizzazione dell'Adriatico, a cura di G. Cuscito (Antichità Altoadriatiche 66), Trieste, 2008, p. 539-551.
} 